\title{
Comorbidity and prognostic indices do not improve the 5-year mortality prediction of components of comprehensive geriatric assessment in hospitalized older patients
}

\author{
Nicolás Martínez-Velilla ${ }^{1 *}$, Koldo Cambra-Contin² and Berta Ibáñez-Beroiz ${ }^{2}$
}

\begin{abstract}
Background: Advancing age is associated with increased vulnerability to chronic health problems. Identifying factors that predict oldest-old status is vital for developing effective clinical interventions and public health strategies.
\end{abstract}

Methods: Observational prospective study of patients aged 75 years and older consecutively admitted to an Acute Geriatric Ward of a tertiary hospital. After a comprehensive geriatric assessment all patients were assessed for five comorbidity indices and two prognostic models. Univariate and multivariate logistic regression models were fitted to assess the association between each score and 5-year mortality. The ability of each score to predict mortality was assessed using the area under the receiver operating characteristic curve.

Results: 122 patients were enrolled. All patients were followed up for five years. 90 (74\%) of them died during the study period. In the logistic regression analyses, apart from age, cognitive impairment and Barthel Index, three indices were identified as statistically associated with 5-year mortality: the Geriatric Index of Comorbidity and the two prognostic indices. The multivariate model that combined age, sex, cognitive impairment and Barthel showed a good discriminate ability (AUC $=0.79$ ), and it did not improve substantially after adding individually any of the indices.

Conclusions: Some prognostic models and the Geriatric Index of Comorbidity are better than other widely used indices such as the Charlson Index in predicting 5-year mortality in hospitalized older patients, however, none of these indices is superior to some components of comprehensive geriatric assessment.

Keywords: 5-year mortality, Aged, Comorbidity scores, Multimorbidity, Comprehensive geriatric assessment

\section{Background}

Advancing age is associated with increased vulnerability to chronic health problems. Identifying factors that predict oldest-old status is vital for developing effective clinical interventions and public health strategies. In clinical practice many decisions are not fully informed unless the patient's prognosis is considered. The strongest and

\footnotetext{
* Correspondence: nicolas.martinez.velilla@cfnavarra.es

'Geriatric Department, Complejo Hospitalario de Navarra, Red de Investigación en Servicios Sanitarios en Enfermedades Crónicas (REDISSEC), Irunlarrea 4, Pamplona 31008, Spain

Full list of author information is available at the end of the article
}

most consistent predictors of mortality in older patients include comorbidity and functional status.

There are several important reasons for measuring comorbidity: to correct for confounding factors, to identify the modification of effects, to predict study outcomes or the natural history of the disease, and finally for statistical efficiency [1]. The terms comorbidity and multimorbidity have been used indistinctly and only in the last few years, a clear distinction between the two terms has been widely accepted [2-4]. Although no "golden standard" for measuring multimorbidity has been established so far, numerous instruments exist, but the issue over which comorbidity index is the best for research purposes is still unclear. A 
range of morbidity scores have been used, of which the Charlson Index (ChI) [5] is the most well known, but evidence for the limitations of this index in older patients is growing [6]. There have been several reviews of prognostic and comorbidity indices for older adults [1,4,7-9]. However, although these indices try to help in the clinical decision-making process, there is still insufficient evidence at this time to recommend the wide-spread use of prognostic indices in clinical practice. Surprisingly, even the more-common models developed some 25 years ago have not been frequently validated in an older subgroup. The applicability of these models in this increasingly important population merits special attention.

Our previous study showed that comprehensive geriatric assessment (CGA) is a better 1-year mortality predictor than several indices and factors [10], and the objective of the present study is to evaluate the long-term prognostic ability of these factors and indices in order to check their eventual role if added to functional and cognitive measures.

\section{Methods}

This is an observational prospective study of patients aged 75 years and older consecutively admitted to an Acute Geriatric Ward of a tertiary hospital in Spain. After a comprehensive geriatric assessment of all the relevant clinical variables. This research was carried out in compliance with the Helsinki Declaration, and informed written consent was obtained from the patient or the next of kin to enter in the study. The assessment included clinical and demographic information, as well as an assessment of dependence in daily activities by means of the Barthel Index [11]. All patients underwent a mental function evaluation using the Spanish version of the Folstein Mini-Mental State Examination [12], with scores ranging from 0 (poor) to 35 (excellent). The same clinical interviewer assessed the following comorbidity and prognostic indices:

\section{Prognostic indices}

The Burden of Illness Score for Elderly Persons (BISEP) by Inouye et al. [13] updated previous indices which had been developed by the same group by adding functional and laboratory data to the diagnoses of administrative data in order to estimate 1-year mortality. Participants were drawn from a prospective study of individuals aged 70 years and older who were hospitalized at Yale-New Haven Hospital. The study was validated in a sample of 1246 participants from 27 Connecticut hospitals who were 65 years and older with a principal discharge diagnosis of pneumonia. The investigators demonstrated improvement in the $\mathrm{C}$ statistic with the addition of laboratory and functional cognitive measures to administrative data.
The Prognostic Index (PI) proposed by Walter et al., [14] was developed to predict 1-year mortality for elderly individuals after hospital discharge using secondary data from a study of patients aged 70 years and older who were hospitalized at the University of Hospitals Cleveland and the Akron City Hospital. Rozzini subsequently externally validated the index's performance predicting 6-month mortality in a retrospective analysis of 840 consecutively admitted participants to a hospital in Italy and found monotonic increases in mortality for each predicted risk level.

\section{Comorbidity indices}

The Charlson Index [5] (ChI) is the most extensively studied comorbidity index. It was originally developed to predict 1-year mortality in 604 patients [5]. It features 19 conditions with assigned weights from 1 to 6 , and a total score is obtained by adding up the weights of all present comorbidity illnesses. The diseases were selected on the basis of the degree of their association with mortality. Although the maximum score is 37 , patients rarely score above 3 (cancer patients).

The Index of Coexistent Disease (ICED) was designed by Greenfield [15] to explain variations in patient health status at hospital admission and discharge, and it is based on the presence and severity of 19 medical conditions and 11 physical impairments, using two subscales: the Individual Disease Severity (IDS) and the Functional Severity (FS). The IDS grades each condition on a 0-4 scale. Scores are based on an explicit list of symptoms, signs, and laboratory tests. Using pre-specified grouping rules, the IDS and FS scores can be combined to create a single score from 0 to 3 . We used the total score, which was categorized into quartiles.

The Diagnosis Count (DC) is a simple and universally available comorbidity index consisting of a numeric sum of all secondary diagnoses and has been demonstrated to be predictive of overall mortality and hospital resource utilization [16,17]. Although this method seems to be quite straightforward, substantial differences exist with regard to the definition used to consider a condition as comorbid.

The Cumulative Illness Rating Scale (CIRS) [18] rates 14 body systems on a five-point severity scale. It was adapted to be used in geriatric populations (CIRS-G) (CIRS geriatric),for which guidelines to enhance reliability have been formulated [19]. Each system is scored as follows: 1 (none)-no impairment to that organ or system; 2 (mild)-impairment does not interfere with normal activity, treatment may or may not be required, prognosis is excellent; 3(moderate)- impairment interferes with normal activity, treatment is needed, prognosis is good; 4 (severe)impairment is disabling, treatment is urgently needed, prognosis is guarded; 5 (extremely severe)- impairment is 
life threatening, treatment is urgent or of no avail, poor prognosis.

The Geriatric index of comorbidity (GIC) [20] classifies patients into 4 classes of increasing somatic comorbidity. The GIC was defined based on information coming from two domains, number of diseases and severity of diseases as measured by Greenfield's IDS. Class I includes patients with one or more conditions with IDS $=1$ or lower. Class II includes patients with one or more conditions with IDS $=2$. Class III includes patients with one condition with IDS $=3$, other conditions having IDS $=2$ or lower. Class IV includes patients with IDS $=3$ or one or more conditions with IDS $=4$.

\section{Outcome measure}

All patients were followed up for five years and assessed in the outpatients clinic or via a telephone interview, and the information was then checked with the computerized clinical history. Then we examined the performance of each comorbidity measure with respect to predicting 5-year mortality after hospital discharge.

\section{Statistical analysis}

Demographic and clinical characteristics of the patients were summarized using descriptive statistics such as frequency and percentages for categorical variables and mean and standard deviation for continuous ones. Scores were categorized into quartiles to facilitate comparisons between indices. Univariate logistic regression models were fitted to assess the association between each score and 5 -years mortality, which provided Odds Ratios (OR) with their 95\% confidence intervals (CI) for the scores' quartiles. Logistic regression analysis models also provided the pseudo $R^{2}$, which was used to compare the percentage of variance explained by each model. The ability of each score to predict mortality was assessed using the area under the receiver operating characteristic curve (AUC), and a value of 0.7 or higher for the AUC was accepted as clinically relevant. Finally, to assess the contribution of each index over components of comprehensive geriatric assessment (age, function, and cognition), a multivariate logistic regression model with the covariates was first fitted, and its AUC was compared with the AUCs obtained by adding independently each one of the scores. Statistical analysis was performed using SPSS for Windows 21.0 (SPSS, Inc., Chicago, IL).

The study was performed in accordance with the Declaration of Helsinki. Informed consent to participate in the study was obtained from all participants or proxies (if the patient had cognitive impairment).

\section{Results}

122 patients were enrolled. Mean age was 85.4 (5.4 SD), $56.6 \%$ were women, $21 \%$ were living in nursing homes, $48.2 \%$ had at least mild cognitive impairment (12.3\% severe) and $62.3 \%$ of them were taking 6 or more drugs. $51.6 \%$ of the patients had a Barthel Index lower than 75 (mean 65.2 SD 34.41). The main diagnoses on admission were acute cardiac and respiratory exacerbations. All patients were followed up during the five years. $90(74 \%)$ of them died during the study period (22\% within the first month and 50\% over the first 4 months). The results of the univariate logistic regression models are given in the left- hand side of Table 1, which shows that apart from age $(\mathrm{p}=0.001)$ and, marginally, Barthel $(\mathrm{p}=0.056)$, the indices GIC $(p=0.012)$, BISEP $(p=0.01)$ and PI $(p=0.005)$ were statistically associated with 5 -year mortality. The risk gradient across quartiles for these three scores is remarkable, as can be picked out from the estimates of the Odds Ratios. The Pseudo $\mathrm{R}^{2}$ statistics was also highest for the univariate models with each of these three indexes $\left(R^{2}=16.1 \%, 17.3 \%\right.$ and $20.9 \%$ respectively). In the same direction, they showed the best predictive ability according to the AUC (0.66, 0.73 and 0.72 respectively), the last two show good predictive ability $(\geq 0.70)$. The multivariate model that combined age, sex, cognitive impairment, and Barthel showed a good discriminate ability (AUC $=0.79$ ), and results did not improve substantially after adding individually any of the indices, as can be seen on the right-hand side of Table 1 .

\section{Discussion}

This study explores the relationship between several comorbidity and prognostic indices and mortality in older hospitalized patients. It identifies which of these indices are more appropriate in the older hospitalized patients and features that components from the comprehensive geriatric assessment (function and cognition) are the cornerstone for prognostic decisions. Our work confirms previous findings that have shown that the Charlson index, used alone, is not the best index in such a complex population [21-25], and adds an innovative approach comparing comorbidity indices and prognostic models, showing that BISEP and PI are useful prognostic tools. The persistence of the same factors to predict 1 and 5-year mortality empower the solidity of our model [10].

Currently, there is no accepted standardized method for measuring and quantifying the prognostic value of comorbid conditions in hospitalized elderly patients with acute disease. Because of competing chronic conditions and diminished life expectancy, careful considerations of prognosis is particularly important for clinical decision making in older patients. It seems that GIC is more suitable in older hospitalized patients as Zekry et al demonstrated [21-23]. They also compared 6 comorbidity indices for one and five-year mortality prediction and found that GIC and CIRS-G were the best predictors. Rozzini [20] did the same in Geriatric Evaluation and Rehabilitation Units for subacute and disabled patients with four indices. 
Table 1 Logistic regression analysis predicting 5-year mortality

\begin{tabular}{|c|c|c|c|c|c|c|}
\hline & & \multicolumn{4}{|c|}{ Univariate logistic regression } & \multirow{2}{*}{$\begin{array}{l}\text { Multivariate } \\
\text { AUC }^{(*)}\end{array}$} \\
\hline & & OR $\left(\mathrm{IC}_{95 \%}\right)$ & $\mathrm{p}$ & $\mathrm{R}^{2}(\%)$ & AUC & \\
\hline \multicolumn{7}{|c|}{ Covariates of adjustment } \\
\hline Age & (Continuous) & $1.17(1.06,1.28)$ & 0.001 & 14.6 & $0.70(0.59,0.81)$ & \\
\hline \multirow[t]{2}{*}{ Sex } & Female & Reference & & & & \\
\hline & Male & $1.17(0.52,2.65)$ & 0.708 & 0.2 & $0.52(0.40,0.64)$ & $0.79(0.71,0.88)$ \\
\hline Cognitive & No & Reference & & & & \\
\hline \multirow[t]{2}{*}{ Impairment } & Mild & $2.02(0.66,6.24)$ & & & & \\
\hline & Mod/severe & $7.58(2.10,27.4)$ & 0.007 & 15.3 & $0.69(0.59,0.79)$ & \\
\hline \multirow[t]{4}{*}{ Barthel } & 4 & Reference & 0.056 & 11.2 & $0.72(0.62,0.82)$ & \\
\hline & 3 & $2.14(0.70,6.58)$ & & & & \\
\hline & 2 & $2.18(0.55,8.68)$ & & & & \\
\hline & 1 & $7.14(1.54,33.15)$ & & & & \\
\hline \multicolumn{7}{|c|}{ Comorbidity indices } \\
\hline \multirow[t]{4}{*}{ Chl } & 1 & Reference & & & & \\
\hline & 2 & $1.92(0.66,5.61)$ & 0.144 & 7.0 & $0.64(0.53,0.75)$ & $0.81(0.73,0.89)$ \\
\hline & 3 & $2.30(0.67,7.90)$ & & & & \\
\hline & 4 & $4.03(1.06,15.31)$ & & & & \\
\hline \multirow[t]{4}{*}{ DC } & 1 & Reference & & & & \\
\hline & 2 & $5.76(1.17,28.24)$ & 0.106 & 8.1 & $0.58(0.45,0.70)$ & $0.80(0.72,0.88)$ \\
\hline & 3 & $2.38(0.84,6.75)$ & & & & \\
\hline & 4 & $1.92(0.66,5.56)$ & & & & \\
\hline \multirow[t]{4}{*}{ CIRS } & 1 & Reference & & & & \\
\hline & 2 & $1.23(0.40,3.78)$ & 0.601 & 2.4 & $0.54(0.42,0.66)$ & $0.80(0.71,0.88)$ \\
\hline & 3 & $0.97(0.34,2.80)$ & & & & \\
\hline & 4 & $2.25(0.60,8.46)$ & & & & \\
\hline \multirow[t]{4}{*}{ ICED } & 1 & Reference & & & & \\
\hline & 2 & $0.70(0.21,2.34)$ & 0.344 & 4.5 & $0.56(0.45,0.67)$ & $0.79(0.71,0.87)$ \\
\hline & 3 & $0.75(0.26,2.12)$ & & & & \\
\hline & 4 & $2.17(0.58,8.20)$ & & & & \\
\hline \multirow[t]{4}{*}{ GIC } & 1 & Reference & & & & \\
\hline & 2 & $0.50(0.15,1.67)$ & 0.012 & 16.1 & $0.66(0.56,0.76)$ & $0.79(0.71,0.88)$ \\
\hline & 3 & $1.04(0.27,4.01)$ & & & & \\
\hline & 4 & $4.62(0.96,22.09)$ & & & & \\
\hline \multicolumn{7}{|c|}{ Prognostic indices } \\
\hline \multirow[t]{4}{*}{ BISEP } & 1 & Reference & & & & \\
\hline & 2 & $1.41(0.52,3.85)$ & 0.010 & 17.2 & $0.73(0.63,0.82)$ & $0.81(0.73,0.89)$ \\
\hline & 3 & $6.92(1.29,37.05)$ & & & & \\
\hline & 4 & $10.0(1.91,52.47)$ & & & & \\
\hline \multirow[t]{4}{*}{ PI } & 1 & Reference & & & & \\
\hline & 2 & $3.10(0.83,11.66)$ & 0.005 & 20.9 & $0.72(0.62,0.83)$ & $0.80(0.71,0.88)$ \\
\hline & 3 & $5.85(1.52,22.57)$ & & & & \\
\hline & 4 & $18.00(4.01,80.71)$ & & & & \\
\hline
\end{tabular}

BISEP: Burden of Illness Score for Elderly Persons; Chl: Charlson Index; CIRS: Cumulative Illness Rating Scale; DC: Disease Count; ICED: Index of Coexistent Disease; GIC: Geriatric Index of Comorbidity; PI: Prognostic Index *Quartile ranges do not aply to ICED and GIC, as these indices are predefined into 4 classes

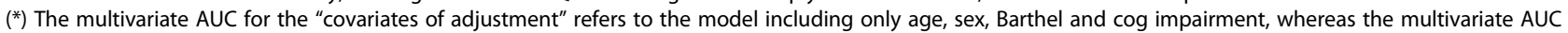
given for each index is the one obtained from the multivariate model including the formers and each one of the indices. 
They found that GIC had the greatest concurrent validity with disability and was the best predictor of mortality. On the other hand, Di Bari et al., [26] found that DC, ChI and ICED performed better than GIC. This was probably because their population was younger (mean age was $74 \pm$ 0.3 ) and less complex than ours and that included in the study of Zekry et al. [21] The GIC captures a significant part of the excess disability due to the co-occurrence of diseases but its hierarchical construction does not allow for the discrimination of degrees of comorbidity conditions whose severity is lower than that of the most severe conditions. It can help clinicians to discriminate and adjust basal status in the different study populations so that a stratification for the analysis can be performed and reliable conclusions be drawn afterwards. None of the $\mathrm{C}$ statistics for the included indices were higher than 0.90, similarly to previous studies that showed $\mathrm{C}$ statistics between 0.662 and 0.77 . This discriminatory ability is consistent with other indices that commonly drive clinical decisions, such as the LACE index (C statistic, 0.684) [27], the TIMI risk score (C statistic, 0.65) [28], the CHADS2 index ( $\mathrm{C}$ statistic, 0.68-0.72) [29] or the Framingham risk score (C statistic, 0.63-0.83) [30].

The results of the current study have also shown that none of the indices improves the prognostic approach of the CGA in predicting survival and adds to the growing evidence that CGA is a better predictor than comorbid conditions, and is able to help to improve care planning, and overall better quality of care. [31-34] Other authors have shown that disability, more than multimorbidity, exerts an important influence on mortality, independently of age and other clinical functional variables [35]. Furthermore, among hospitalized elderly subjects, functional impairment rather than comorbidity and indices of disease burden have been recognized as the strongest predictors of outcomes [36-38]. The Barthel Index could therefore be more useful than other indexes when considering an adequate use of healthcare services [24]. Cognitive impairment is the other pillar of this model, and it has been validated as an excellent predictor of adverse outcomes.

We have to analyze these conclusions in a comprehensive geriatric context. Even if function and cognition are the main long term mortality predictors, we should always consider other areas like comorbidity, social support or age. As a matter of fact, function and mental status could be recognized as the background defining elderly health status and help to stratify a spectrum of alternative therapeutic approaches, from life saving to palliative and symptomatic care. All these issues are complementary and not mutually exclusive.

The main strength of this study is the prospective comprehensive assessment of several indices and prognostic models of mortality. As far as we know, this is the first prospective study comparing at the same time, comorbidity indices, prognostic models and CGA for the prediction of 5-year mortality. Previous studies have used only few or just one index and have been done in a community-dwelling population, and only a few of them present the coefficient of determination $\left(R^{2}\right)$ of their model $[9,20,39]$. Our study is difficult to generalize because the data are obtained in a small sample of hospitalized older patients with a high burden of comorbidities, disability and cognitive impairment.

\section{Conclusions}

In conclusion, BISEP, PI and GIC indices are better than other widely used indices such as $\mathrm{ChI}$ in predicting 5 -years mortality in hospitalized older patients, but none of these indices are superior to some components of CGA. The evidence that function and cognition have a greater effect than multimorbidity on mortality prediction suggests moving from a disease-centered model for frail older patients to an integrative cognitive and functional management.

\section{Abbreviations}

BISEP: Burden of illness score for elderly persons; CGA: Comprehensive geriatric assessment; CIRS: Cumulative illness rating scale; Chl: Charlson index; DC: Diagnosis count; GIC: Geriatric index of comorbidity; ICED: Index of coexistent disease; IDS: Individual disease severity; PI: Prognostic index.

\section{Competing interest}

The authors declare that they have no competing interests.

\section{Authors' contributions}

NM carried out the conception, design and acquisition of data, and wrote the manuscript. KC carried out the statistical analysis and helped to draft the manuscript. BI carried out the statistical analysis and helped to draft the manuscript. All authors read and approved the final manuscript.

\section{Acknowledgements}

We would like to thank all participants, their families and their primary care teams for their participation in the study. We also wish to thank Dr Miguel Andériz López for his helpful comments during the development of Dr Martinez Velilla PhD thesis, and Raúl Azpilicueta Martínez for his help with language editing.

\section{Author details}

'Geriatric Department, Complejo Hospitalario de Navarra, Red de Investigación en Servicios Sanitarios en Enfermedades Crónicas (REDISSEC), Irunlarrea 4, Pamplona 31008, Spain. ${ }^{2}$ Navarrabiomed-Fundación Miguel Servet, Red de Investigación en Servicios Sanitarios en Enfermedades Crónicas (REDISSEC), Pamplona, Spain.

Received: 23 December 2013 Accepted: 25 April 2014

Published: 15 May 2014

\section{References}

1. de Groot V, Beckerman H, Lankhorst GJ, Bouter LM: How to measure comorbidity. a critical review of available methods. J Clin Epidemiol 2003, $56: 221-229$.

2. Fortin M, Stewart M, Poitras ME, Almirall J, Maddocks H: A systematic review of prevalence studies on multimorbidity: toward a more uniform methodology. Ann Fam Med 2012, 10:142-151.

3. van den Akker MBF, Knottnerus JA: Comorbidity or multimorbidity: what's in a name? A review of literature. Eur J Gen Pract 1996, 2:65-70.

4. Martinez Velilla NI, Gaminde Inda ID: [Comorbidity and multimorbidity indexes in the elderly patients]. Med Clin (Barc) 2011, 136:441-446. 
5. Charlson ME, Pompei P, Ales KL, MacKenzie CR: A new method of classifying prognostic comorbidity in longitudinal studies: development and validation. J Chronic Dis 1987, 40:373-383.

6. Min L, Wenger N, Walling AM, Blaum C, Cigolle C, Ganz DA, Reuben D, Shekelle P, Roth C, Kerr EA: When comorbidity, aging, and complexity of primary care meet: development and validation of the Geriatric CompleXity of Care Index. J Am Geriatr Soc 2013, 61:542-550.

7. Diederichs $C$, Berger $K$, Bartels DB: The measurement of multiple chronic diseases-a systematic review on existing multimorbidity indices. J Gerontol A Biol Sci Med Sci 2011, 66:301-311.

8. Hall SF: A user's guide to selecting a comorbidity index for clinical research. J Clin Epidemiol 2006, 59:849-855.

9. Harboun M, Ankri J: [Comorbidity indexes: review of the literature and application to studies of elderly population]. Rev Epidemiol Sante Publique 2001, 49:287-298.

10. Martinez-Velilla N, Ibanez-Beroiz B, Cambra-Contin K, Alonso-Renedo J: Is comprehensive geriatric assessment a better 1-year mortality predictor than comorbidity and prognostic indices in hospitalized older adults? J Am Geriatr Soc 2013, 61:1821-1823.

11. Mahoney Fl, Barthel DW: Functional evaluation: the barthel index. Md State Med J 1965, 14:61-65.

12. Lobo AEJ, Gómez FB, Sala JM, Seva A: El Mini Examen cognoscitivo. Un test sencillo y práctico para detectar alteraciones intelectuales en pacientes médicos. Actas Luso-Esp Neurol Psiquiatr 1979, 7:189-202.

13. Inouye SK, Bogardus ST Jr, Vitagliano G, Desai MM, Williams CS, Grady JN Scinto JD: Burden of illness score for elderly persons: risk adjustment incorporating the cumulative impact of diseases, physiologic abnormalities, and functional impairments. Med Care 2003, 41:70-83.

14. Walter LC, Brand RJ, Counsell SR, Palmer RM, Landefeld CS, Fortinsky RH, Covinsky KE: Development and validation of a prognostic index for 1-year mortality in older adults after hospitalization. JAMA 2001 285:2987-2994.

15. Greenfield S, Sullivan L, Dukes KA, Silliman R, D'Agostino R, Kaplan SH: Development and testing of a new measure of case mix for use in office practice. Med Care 1995, 33:AS47-AS55.

16. Melfi C, Holleman E, Arthur D, Katz B: Selecting a patient characteristics index for the prediction of medical outcomes using administrative claims data. J Clin Epidemiol 1995, 48:917-926.

17. Satariano WA, Ragland DR: The effect of comorbidity on 3-year survival of women with primary breast cancer. Ann Intern Med 1994, 120:104-110.

18. Linn BS, Linn MW, Gurel L: Cumulative illness rating scale. J Am Geriatr SoC $1968,16: 622-626$

19. Salvi F, Miller MD, Grilli A, Giorgi R, Towers AL, Morichi V, Spazzafumo L, Mancinelli L, Espinosa E, Rappelli A, Dessi-Fulgheri P: A manual of guidelines to score the modified cumulative illness rating scale and its validation in acute hospitalized elderly patients. J Am Geriatr Soc 2008, 56:1926-1931.

20. Rozzini R, Frisoni GB, Ferrucci L, Barbisoni P, Sabatini T, Ranieri P, Guralnik JM, Trabucchi M: Geriatric index of comorbidity: validation and comparison with other measures of comorbidity. Age Ageing 2002 31:277-285.

21. Zekry D, Loures Valle BH, Graf C, Michel JP, Gold G, Krause KH, Herrmann FR: Prospective comparison of 6 comorbidity indices as predictors of 1-year post-hospital discharge institutionalization, readmission, and mortality in elderly individuals. J Am Med Dir Assoc 2012, 13:272-278.

22. Zekry D, Loures Valle BH, Lardi C, Graf C, Michel JP, Gold G, Krause KH, Herrmann FR: Geriatrics index of comorbidity was the most accurate predictor of death in geriatric hospital among six comorbidity scores. J Clin Epidemiol 2010, 63:1036-1044.

23. Zekry D, Valle BH, Michel JP, Esposito F, Gold G, Krause KH, Herrmann FR: Prospective comparison of six co-morbidity indices as predictors of 5 years post hospital discharge survival in the elderly. Rejuvenation Res 2010, 13:675-682

24. Torres Moreno B, Nunez Gonzalez E, Perez Hernandez Dde G, Simon Turriate JP, Alastuey Gimenez C, Diaz Melian J, Corujo Rodriguez E, Gonzalez Bermudez MD, Fernandez Duque O: [Barthel and Charlson indexes for the prognosis of mortality and institutionalization in hospitalized geriatric patients]. Rev Esp Geriatr Gerontol 2009, 44:209-212.

25. Testa G, Cacciatore F, Galizia G, Della-Morte D, Mazzella F, Russo S, Ferrara N, Rengo F, Abete P: Charlson comorbidity index does not predict long-term mortality in elderly subjects with chronic heart failure. Age Ageing 2009, 38:734-740.
26. Di Bari M, Virgillo A, Matteuzzi D, Inzitari M, Mazzaglia G, Pozzi C, Geppetti P, Masotti G, Marchionni N, Pini R: Predictive validity of measures of comorbidity in older community dwellers: The Insufficienza Cardiaca negli Anziani Residenti a Dicomano Study. J Am Geriatr Soc 2006, 54:210-216

27. van Walraven C, Dhalla IA, Bell C, Etchells E, Stiell IG, Zarnke K, Austin PC, Forster AJ: Derivation and validation of an index to predict early death or unplanned readmission after discharge from hospital to the community. CMAJ 2010, 182:551-557.

28. Antman EM, Cohen M, Bernink PJ, McCabe CH, Horacek T, Papuchis G, Mautner B, Corbalan R, Radley D, Braunwald E: The TIMI risk score for unstable angina/non-ST elevation MI: a method for prognostication and therapeutic decision making. JAMA 2000, 284:835-842.

29. Rietbrock S, Heeley E, Plumb J, van Staa T: Chronic atrial fibrillation: Incidence, prevalence, and prediction of stroke using the Congestive heart failure, Hypertension, Age $>75$, Diabetes mellitus, and prior Stroke or transient ischemic attack (CHADS2) risk stratification scheme. Am Heart J 2008, 156:57-64.

30. D'Agostino RB Sr, Grundy S, Sullivan LM, Wilson P: Validation of the Framingham coronary heart disease prediction scores: results of a multiple ethnic groups investigation. JAMA 2001, 286:180-187.

31. Chen L-K, Peng L-N, Lin M-H, Lai H-Y, Hwang S-J, Lan C-F: Predicting mortality of older residents in long-term care facilities: comorbidity or care problems? J Am Med Dir Assoc 2010, 11:567-571.

32. Taekema DG, Gussekloo J, Westendorp RGJ, de Craen AJM, Maier AB: Predicting survival in oldest old people. Am J Med 2012, 125:1188-1194. e1181.

33. Rockwood K, Howlett S, Stadnyk K, Carver D, Powell C, Stolee P. Responsiveness of goal attainment scaling in a randomized controlled trial of comprehensive geriatric assessment. J Clin Epidemiol 2003, 56:736-743.

34. Landi F, Onder G, Russo A, Tabaccanti S, Rollo R, Federici S, Tua E, Cesari M, Bernabei R: A new model of integrated home care for the elderly: impact on hospital use. J Clin Epidemiol 2001, 54:968-970.

35. Landi F, Liperoti R, Russo A, Capoluongo E, Barillaro C, Pahor M, Bernabei R, Onder G: Disability, more than multimorbidity, was predictive of mortality among older persons aged 80 years and older. In J Clin Epidemiol, Volume 63. United States: Elsevier Inc; 2010:752-759.

36. Fried LP, Bandeen-Roche K, Kasper JD, Guralnik JM: Association of comorbidity with disability in older women: the Women's Health and aging study. J Clin Epidemiol 1999, 52:27-37.

37. Inouye SK, Peduzzi PN, Robison JT, Hughes JS, Horwitz RI, Concato J: Importance of functional measures in predicting mortality among older hospitalized patients. JAMA 1998, 279:1187-1193.

38. Incalzi RA, Capparella O, Gemma A, Landi F, Bruno E, Di Meo F, Carbonin P The interaction between age and comorbidity contributes to predicting the mortality of geriatric patients in the acute-care hospital. $J$ Intern Med 1997, 242:291-298.

39. Rozzini R, Sabatini T, Barbisoni P, Trabucchi M: How to measure comorbidity in elderly persons. J Clin Epidemio/ 2004, 57:321-322. author reply 323.

\section{doi:10.1186/1471-2318-14-64}

Cite this article as: Martínez-Velilla et al:: Comorbidity and prognostic indices do not improve the 5-year mortality prediction of components of comprehensive geriatric assessment in hospitalized older patients. BMC Geriatrics 2014 14:64 